\title{
DIFFUSION OF OXYGEN VACANCIES IN YTTRIUM IRON GARNET INVESTIGATED BY DYNAMIC CONDUCTIVITY MEASUREMENTS
}

\author{
R. MetselaAR and P. K. LARSEN \\ Philips Research Laboratories, Eindhoven, The Netherlands
}

(Received 10 July 1975; accepted 31 October 1975)

\begin{abstract}
In yttrium iron garnet, $\mathrm{Y}_{3} \mathrm{Fe}_{5} \mathrm{O}_{12}$, the oxygen vacancy concentration at high temperatures depends on the partial oxygen pressure. Due to the electron donating nature of the vacancies, changes in the oxygen vacancy concentration can be measured by electrical conductivity measurements. We discuss a dynamic method for studying the diffusion of oxygen vacancies by measurements of the time dependence of the electrical conduction after a change in the oxygen partial pressure has taken place. It is shown that the interpretation of the measurements is straightforward if the relative change in conductivity remains small $(\leqslant 10 \%)$. Measurements were performed on single crystals and on polycrystalline samples at temperatures $900-1400^{\circ} \mathrm{C}$. The samples were made $n$-type by substitution with $\mathrm{Si}$ or $p$-type by substitution with $\mathrm{Ca}, \mathrm{Zn}$ or $\mathrm{Pb}$. The partial oxygen pressure was changed between 1 and $0.1 \mathrm{~atm}$. For all samples the diffusion coefficient $D$ of the oxygen vacancies can be represented by $D=A \exp (-Q / k T)$, where $A=8400 \mathrm{~cm}^{2} \mathrm{~s}^{-1}$ and $Q=2.90 \mathrm{eV}$. It is shown that the activation energy of $2.90 \mathrm{eV}$ is due to the migration enthalpy of the vacancies only.
\end{abstract}

\section{INTRODUCTION}

Until recently native point defects were mostly neglected in the study of the ferrimagnetic oxide $\mathrm{Y}_{3} \mathrm{Fe}_{5} \mathrm{O}_{12}$, yttrium iron garnet (YIG). The presence of native defects strongly influences the electrical, optical $[1,2]$ and magnetic properties [3]. More specifically the magnitude of the photomagnetic effect, that is the change in the magnetic permeability upon irradiation, was found to be proportional to the concentration of oxygen vacancies [3]. The annealing behaviour of the growth-induced anisotropy in substituted iron garnets used in bubble domain devices is also dependent on the diffusion rate of oxygen vacancies [4]. A study of the oxygen diffusion rate is therefore of great importance for control of the annealing treatments of iron garnets.

In an earlier investigation we described the influence of the partial oxygen pressure at high temperatures on the electrical conductivity of YIG[5]. It was shown that a lowering of the oxygen partial pressure produced an increase in the concentrations of native donors which can be oxygen vacancies or interstitial metal ions. The presence of oxygen vacancies in YIG was demonstrated by Paladino et al.[6] who carried out tracer diffusion experiments of $\mathrm{O}^{18}$ under a constant partial pressure of oxygen. Due to the large ratio of the anion to cation radii the probability of oxygen interstitials is very low, and the diffusion of the tracer atoms $\mathrm{O}^{18}$ in YIG can therefore only be achieved by jumps via vacant oxygen sites. Thermogravimetric measurements in YIG show that at high temperatures a reversible weight change takes place when the partial oxygen pressure $P_{\mathrm{O}_{2}}$ is changed[7]. Following the atomic notation of Kröger and Vink the creation or destruction of $m$-fold positively charged oxygen vacancies $V_{0}{ }^{m} \cdot(m=0,1,2)$ can be described by the reaction equation

$$
\mathrm{O}_{0} \rightleftarrows V_{0}^{m \cdot}+m e^{\prime}+\frac{1}{2} \mathrm{O}_{2}(g)
$$

$\mathrm{O}_{0}$ denotes oxygen ions on a normal lattice site, $V_{0}$ are oxygen vacancies, $e$ are electrons, $\mathrm{O}_{2}(g)$ denotes gaseous oxygen molecules and the dots and dashes indicate positive and negative charges relative to the normal charge of the neutral lattice.

Let us next consider the possibility of interstitial $Y$ and/or $\mathrm{Fe}$ ions. If the reversible weight change mentioned above is to be explained in terms of metal interstitials $Y_{i}^{s}$. and $\mathrm{Fe}_{i}{ }^{\nu}(s, \nu=0,1,2,3)$ we have to consider the reaction

$$
12 \mathrm{O}_{0} \rightleftarrows 3 Y_{i}^{s}+5 \mathrm{Fe}_{i}{ }^{\nu}+(3 s+5 v) e^{\prime}+6 \mathrm{O}_{2}(g) .
$$

Interstitial yttrium and irons ions have to be formed in the ratio 3:5 when atoms are removed from the lattice. We have calculated that the garnet structure (space group $O_{h}{ }^{10}$ ) can accommodate interstitial ions with radii less than $0.8 \AA$ on the empty $b$-sites. This means that the small iron ions are allowed here, but that the probability for interstitial yttrium ions is very low. We therefore consider variations in donor concentration due to interstitials unlikely and in the following we shall assume that, in YIG, oxygen vacancies are the only native point defects acting as donor. We found no indications that native defects with acceptor properties are present.

There is evidence that at room temperature or below the electrons $e^{\prime}$ can be localized on the iron ions, i.e. we have to write $\mathrm{Fe}^{\prime}$ instead of $e^{\prime}$. We have indeed used this notation in Refs. [1,3]. At the high temperature processes discussed in this paper $\left(T>900^{\circ} \mathrm{C}\right)$ the conduction seems to be well described in terms of transport of charge carriers in the valence or conduction bands [8]. Therefore, and without losing generality we write $e^{\prime}$ and $h^{\circ}$, where in some cases $\mathrm{Fe}^{\prime}$ or $\mathrm{Fe}^{\cdot}$ (i.e. in the ionic notation $\mathrm{Fe}^{2+}$ or $\mathrm{Fe}^{4+}$ respectively) could be written.

Application of the law of mass action to eqn (1) then gives

$$
\left[V_{0}^{m} \cdot\right] n^{m}=K_{v}(T) P_{0_{2}}^{-1 / 2}
$$


where the brackets denote the concentration of oxygen vacancies, $n=\left[e^{\prime}\right]$ is the electron concentration and $K_{v}(T)$ is the equilibrium constant of this reaction. Equation (3) shows the dependence of the oxygen vacancy concentration on the oxygen partial pressure. Due to the electrical donor nature of these defects the time dependence of the change towards a new equilibrium state can be obtained by measuring the rate of change of the electrical conductivity after a stepwise change in $P_{\mathrm{O}_{2}}$. The diffusion rate of the oxygen vacancies can be evaluated by means of this dynamic method. We have used this technique to investigate the diffusion rate in single crystals and in polycrystalline samples of YIG of both $n$ - and $p$-type. The theory of this measuring method is discussed in Section 2. Subsection 2(a) gives the solution of the diffusion equation for a thin slab, together with the conditions which have to be fulfilled when we use these equations to ohtain the diffusion constant from electrical conductivity measurements. In Subsection 2(b) and the associated appendix we discuss how far the forementioned conditions are satisfied for a partially compensated semiconducting material like YIG. Details of the measuring cell and information about the samples is given in Section 3. Finally, in Sections 4 and 5 the experimental data are given and the results compared with literature on $\mathrm{O}^{18}$ tracer diffusion experiments.

\section{MEASURING METHOD}

(a) Diffusion and conductivity in a thin slab

A useful sample geometry for which the diffusion equations can be solved is that of a thin slab, i.e. a parallelepiped with two sides much greater than the third. We consider a plane sheet of thickness 21 bounded by the planes $x= \pm 1$ (Fig. 1). We assume that the effective (i.e. measured) diffusion coefficient $\tilde{D}$ of the diffusing species
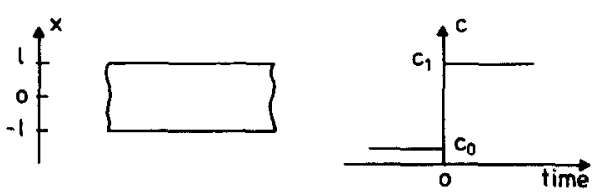

Fig. 1. One-dimensional plate with boundaries at $x= \pm 1$.

is independent of the concentration of these; furthermore that at time $t<0$ the region $-1<x<1$ is at a uniform concentration $C_{0}$ and that the surfaces for times $t \geqslant 0$ are kept at a constant concentration $C_{1}$. The concentration $C=C(x, t)$ at place $x$ and time $t$ is than given by [9]

$$
\begin{aligned}
\frac{C-C_{0}}{C_{1}-C_{0}}= & 1-\frac{4}{\pi} \sum_{k=0}^{\infty} \frac{(-1)^{k}}{2 k+1} \\
& \times \exp \left(-\tilde{D}(2 k+1)^{2} \pi^{2} t / 4 l^{2}\right) \cos \frac{(2 k+1) \pi x}{2 l} .
\end{aligned}
$$

If the relation between the electrical conductivity $\sigma(x, t)$ and the defect concentration $C(x, t)$ is given by $\sigma=f(C)$ or by $C=\psi(\sigma)$ we can determine the diffusion coefficient from the conductivity. A condition implicitly assumed is that in the concentration and conductivity range studied the functions $f$ and $\psi$ are monotonic and continuous ( $\psi=f^{-1}$ ). The conductance $G=G(t)$ parallel to the plane of the sheet is per unit area given by

$$
G(t)=\int_{-1}^{+l} \sigma(x, t) \mathrm{d} x=\int_{-1}^{+l} f(C(x, t)) \mathrm{d} x
$$

The function $f$ depends on the actual material properties as defined by the equilibrium constants for all reaction equations of the species involved. In Subsection 2(b) we shall discuss some different cases. Except for the case of linear relationship between $\sigma$ and $C$, which is discussed below, an iterative procedure is necessary to determine the diffusion coefficient by means of eqns (4) and (5).

In the remainder of this section we consider the special case in which there is a linear dependence of the conductivity $\sigma$ on the defect concentration $C$, i.e.

$$
\sigma(C)=\sigma\left(C_{0}\right)+A C
$$

where $A$ is a constant.

Equations (4) and (5) then reduce to

$$
\begin{aligned}
\frac{G-G_{0}}{G_{1}-G_{0}} & =\int_{-1}^{+1} \frac{C-C_{0}}{C_{1}-C_{0}} \mathrm{~d} x \\
& =1-\sum_{k=0}^{\infty} \frac{8}{(2 k+1)^{2} \pi^{2}} \exp \left(-\tilde{D}(2 k+1)^{2} \pi^{2} t / 4 l^{2}\right)
\end{aligned}
$$

This solution can be approximated for two limiting cases. Using the dimensionless parameter $\theta=D t / 4 l^{2}$ we get for "small times", $\theta \leqslant 0.08$,

$$
\left(G-G_{0}\right) /\left(G_{1}-G_{0}\right)=4(\theta / \pi)^{1 / 2}
$$

while for "long times", $\theta \geqslant 0.035$

$$
\left(G-G_{0}\right) /\left(G_{1}-G_{0}\right)=1-\frac{8}{\pi^{2}} \exp \left(-\pi^{2} \theta\right) .
$$

The two expressions, eqns (8) and (9) approximate the correct solution of eqn (7) within $1 \%$ for the indicated $\theta$ ranges. It should be noted that there is a considerable overlap of the $\theta$-values given. The experimental determination of $\tilde{D}$ from the time dependence of the conductivity is straightforward if eqn (6) is applicable. For small times the conductance varies as $t^{1 / 2}$ and from the slope of $\left(G-G_{0}\right)$ vs $t^{1 / 2} \tilde{D}$ can be determined. For long times $\tilde{D}$ can be found from the slope of a plot of $\ln \left(G_{1}-G\right)$ vs $t$.

It can be shown easily that the approximation of the sample by a thin slab, viz. the use of the one-dimensional expressions (4) and (7) gives a value of $\tilde{D}$ which is correct within $1 \%$ when the length: thickness ratio exceeds ten.

\section{(b) The relation between the conductivity and the native defect concentration}

It has been shown above (Section 2a) that the interpretation of dynamic conductivity measurements is straightforward if a linear relationship between the conductivity and the diffusing defects exists. A solution is also possible if the function $f(C)$ is known, however, for YIG, as for many other compounds, we do not yet have such knowledge. We therefore have to investigate 
experimental conditions under which the relationship $\sigma=\sigma(C)$ between $\sigma$ and $C$ as a good approximation can be considered as linear. Using a Taylor expansion we can write

$$
\sigma(C)=\sigma\left(C_{0}\right)+\sigma^{(1)}\left(C_{0}\right)\left(C-C_{0}\right)+\frac{1}{2 !} \sigma^{(2)}\left(C_{0}\right)\left(C-C_{0}\right)^{2}
$$

where $\sigma^{(n)}=\partial^{n} \sigma / \partial C^{n}$. We see that by kecping the change in the concentration of the diffusing species sufficiently small, the first order term becomes the dominant term, i.e. the relationship can be considered as linear.

In the Appendix we have more specifically investigated the conditions for linearization. We show there, that if the defects, foreign as well as native, are fully ionized, we have a strictly linear relationship as long as $p \gg n$ or $p \ll n$. Where there is incomplete ionization of the defects we find that by keeping $\left[\sigma(C)-\sigma\left(C_{0}\right)\right] / \sigma\left(C_{0}\right)<0.1$ the error using eqn (7) under our typical experimental conditions can be estimated to be smaller than $6 \%$.

If the relative change of $C$ is too large and the ionization is incomplete, the diffusion curves will not be symmetrical with respect to increasing or decreasing defect concentration[10].

\section{EXPERIMENTAL}

Diffusion measurements have been performed on $n$-type as well as $p$-type single crystals and on a $p$-type polycrystalline sample. All crystals were grown from a $\mathrm{PbO}-\mathrm{B}_{2} \mathrm{O}_{3}-\mathrm{PbF}_{2}$ flux. The $n$-type crystals were obtained by substituting small amounts of iron $\left(\mathrm{Fe}^{3+}\right)$ by silicon $\left(\mathrm{Si}^{4+}\right)$; the $p$-type crystals by zinc $\left(\mathrm{Zn}^{2+}\right)$ substitution. It is well known that lead ions from the flux are always incorporated in the crystals. The $\mathrm{Pb}^{2+}$ ions substitute for yttrium $\left(\mathrm{Y}^{3+}\right)$ and act as acceptors. In some cases traces of fluorine are found which have to be considered as electron donors. The result of a chemical analysis for a $\mathrm{Zn}$ doped and for two Si doped single crystals are given in Table 1. $\mathrm{Pb}, \mathrm{Ca}$ and $\mathrm{Zn}$ were determined from atomic absorption measurements, for $\mathrm{Si}$ and $\mathrm{F}$ a photometric analysis was used [11].

The polycrystalline samples used for the diffusion measurements were made $p$-type by substitution of about 0.04 yttrium ions per formula unit by $\mathrm{Ca}$ ions. These samples were produced by sintering of spray dried powders, using sulphates as starting materials.

The results of a spectrochemical analysis are also given in Table 1. For the measurements of the electrical conductivity samples were used with typical dimensions $8 \times 5 \times 0.4 \mathrm{~mm}^{3}$. The single crystal slices were sawn parallel to the (110) growth facets.
This size ensures that the one-dimensional diffusion equations (see Section 2(a)) can be applied.

To remove surface layers the samples were etched in boiling $\mathrm{HCl}$ and rinsed with water after sawing. The end faces were then covered with layers of sputtered platinum of about $0.5 \mu \mathrm{m}$ thickness to obtain ohmic contacts for measurements in the temperature region $300-1400^{\circ} \mathrm{C}$. The sample is placed between spring-loaded Pt-contacts inside an alumina tube, which in turn is placed inside a high tcmperature SiC furnacc. In Fig. 2 a simplified drawing of the measuring cell is shown. All alumina parts are made of gas-tight, highresistivity alumina. The Pt-contacts at the ends of the sample are both connected to the outside of the tube by a Pt-wire for the current supply and by a $\mathrm{Pt} / \mathrm{Pt}-10 \% \mathrm{Rh}$ thermocouple which was used both for voltage and temperature measurements. A gas flow is maintained through the inlet tube which has an aperture below the sample. The dimensions and geometry of the measuring cell have been chosen so that the gas volume in the cell is minimal and that after a change of the oxygen partial pressure of the gas streaming through the inlet tube the new equilibrium pressure at the sample could be reached in as short a time as possible. Our experimental results indicate that by switching the $\mathrm{O}_{2}$ pressure between 1 and $0.1 \mathrm{~atm}$, which is a typical range used for our diffusion measurements, the equilibration pressure at the sample is established within 1 sec. The partial oxygen pressure in the gas is measured by means of zirconia oxygen gauges connected to the inlet and outlet flanges of the cell.

\section{EXPERIMENTAL RESULTS}

An extensive investigation of the temperature dependence of the diffusion coefficient of oxygen vacancies has been performed on a $\mathrm{Zn}$ doped single crystal of YIG which shows $p$-type conduction. Furthermore, results on three other samples of YIG will be compared with the results on the $\mathrm{Zn}$-doped crystal.

In Fig. 3 we show the time dependence of the

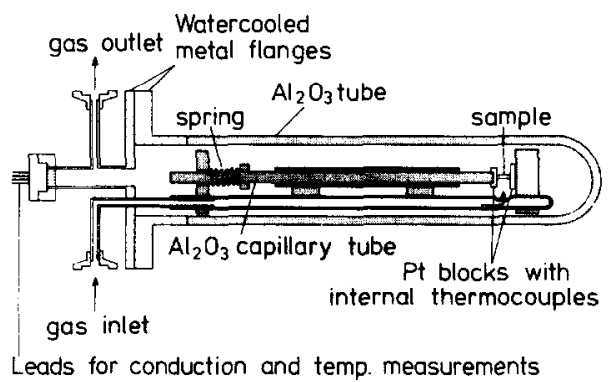

Fig. 2. Schematic drawing of the measuring cell used for dynamic conductivity measurements.

Table 1. Chemical analysis of the extrinsic acceptors $\mathrm{Pb}, \mathrm{Ca}, \mathrm{Zn}$ and donors $\mathrm{Si}, \mathrm{F}$ in single crystals of yttrium iron garnet

\begin{tabular}{l|c|c|c|c|c|c} 
& \multicolumn{3}{|c|}{ Concentrations in molar fractions } \\
& $\mathrm{Pb}$ & $\mathrm{Ca}$ & $\mathrm{Zn}$ & $\mathrm{Si}$ & $\mathrm{F}$ \\
\hline P-type sample, polycrystalline & - & 0.020 & - & 0.002 & - \\
P-type sample, single crystal & 0.0081 & 0.00007 & 0.0122 & 0.0005 & $\ldots$ \\
N-type sample, single crystal A & 0.022 & 0.0014 & - & 0.027 & 0.008 \\
N-type sample, single crystal B & 0.023 & 0.0017 & - & 0.059 & 0.001 \\
\hline
\end{tabular}




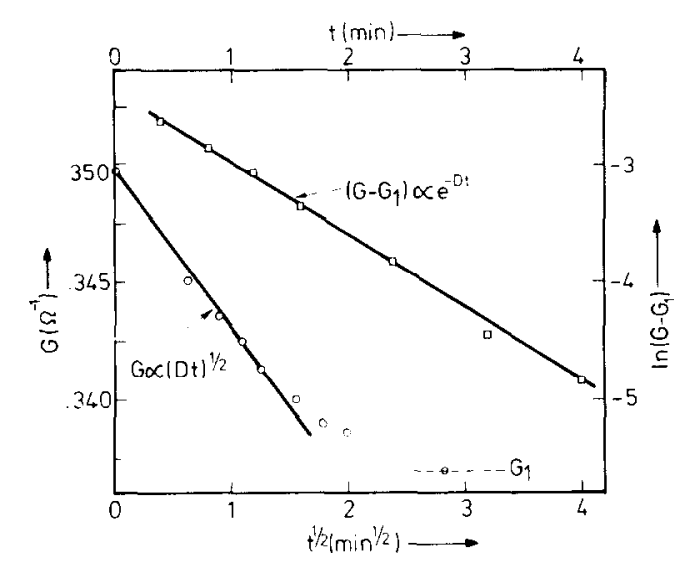

Fig. 3. Time dependence of the conductance $G$ of a single crystalline Zn-doped YIG sample after a change of the partial oxygen pressure at $t=0$ from 1 to $0.1 \mathrm{~atm}$ at $1241^{\circ} \mathrm{C}$. From the initial stage with $G \propto \sqrt{ } t$, we find $D=1.46 \times 10^{-6} \mathrm{~cm}^{2} \mathrm{~s}^{-1}$, from the final stage with $\ln \left(G-G_{1}\right) \propto t$, we find $D=1.49 \times 10^{-6} \mathrm{~cm}^{2} \mathrm{~s}^{-1}$. Here $G_{1}$ is the equilibrium conduction value at $0.1 \mathrm{~atm}$.

conductance of the sample after a change in the partial oxygen pressure from 1 to $0.1 \mathrm{~atm}$ at $1241^{\circ} \mathrm{C}$. For small times $t$ we see that $G \propto \sqrt{ } t$ and for long times $\ln \left(G-G_{1}\right) \propto t$, i.e. the relationships given by eqns $(8)$ and (9) are obtained. The values of the diffusion coefficient calculated from these two approximations agree well with each other. The relative difference of the equilibrium values of the conduction at the two partial pressures used is further seen to be less than $4 \%$. This means that the conduction varies in good approximation linearly with the oxygen vacancy concentration (see Section 2).

Using the experimentally determined value of $\tilde{D}_{v}$ we can calculate the time dependence of $G$ and compare it with the measured curve. Such a comparison is shown in Fig. 4. The overall agreement is seen to be good, which shows that eqn (7) is obeyed indeed.

Our results for the $\mathrm{Zn}$-doped crystal are shown in Fig. 5 . The data can be represented by an equation of the form

$$
\tilde{D}_{r}=A \exp (-\tilde{Q} / k T)
$$

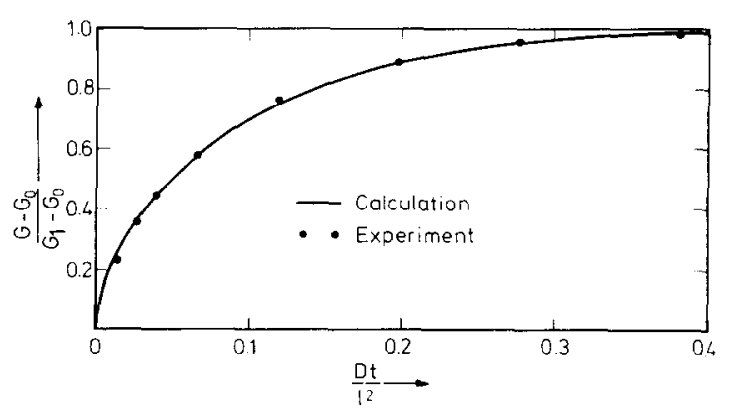

Fig. 4. Dependence of the relative conductance $\left(G-G_{0}\right) /\left(G_{1}-\right.$ $G_{0}$ ) of the dimensionless parameter $D t / l^{2}$, for a single-crystalline $\mathrm{Zn}$-doped YIG sample after a stepwise change of the partial oxygen pressure at $t=0$ from 0.1 to $1 \mathrm{~atm}$ at $1290^{\circ} \mathrm{C}$. $G$ is the conductance at time $t, G_{0}$ is the equilibrium value at $0.1 \mathrm{~atm}, G_{1}$ is the equilibrium value at $1 \mathrm{~atm}, D$ is the diffusion constant, $l$ is half the thickness of a thin slab (actually $2 l=0.4 \mathrm{~mm}$, other dimensions $8 \times 5 \mathrm{~mm}^{2}$ ). Solid line: calculated values (see text), dots: experimental data points.

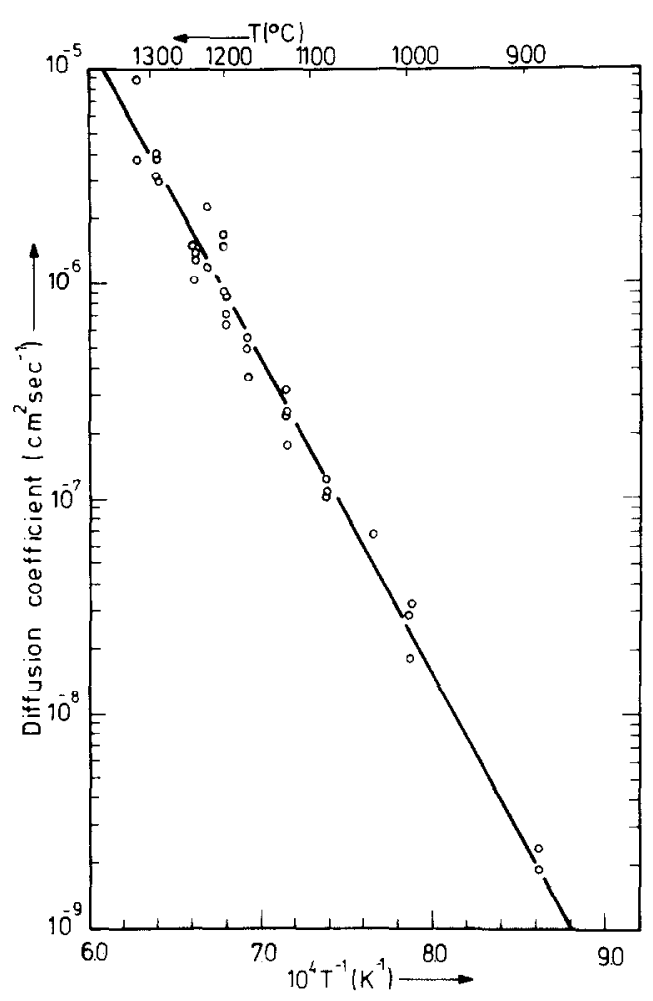

Fig. 5. Circles: Measured values of the oxygen vacancy diffusion coefficient $\tilde{D}$ for a single crystalline $\mathrm{Zn}$-doped YIG crystal. Line: Least squares fit through the data points, $\tilde{D}=$ $8400 \exp (-\tilde{Q} / k T) \mathrm{cm}^{2} \mathrm{~s}^{-1}$, with $\tilde{Q}=2.90 \pm 0.10 \mathrm{eV}$.

The straight line in this figure is a least squares fit through the data points with $A=(8400 \pm 800) \mathrm{cm}^{2} \mathrm{~s}^{-1}$ and $\tilde{Q}=$ $(2.90 \pm 0.10) \mathrm{eV}$.

The results of the measurements on two different Si-substituted crystals which have $n$-type conduction and on a Ca-substituted $p$-type polycrystalline sample are shown in Fig. 6. For comparison with the results obtained on the Zn-substituted crystal we have drawn the curve given by eqn (11). We see that this equation gives a reasonable representation of the diffusion coefficient for all four samples which have been measured.

The diffusion studied in this section has taken place under a concentration gradient and the diffusion coefficient obtained in this way is therefore the chemical diffusion coefficient. For an interpretation of our experimental results we consider in the next section the meaning of $\tilde{D}_{\mathrm{v}}$ in more detail.

\section{DISCUSSION}

(a) The chemical and microscopic diffusion coefficient

We assume that the oxygen vacancy diffusion in the garnet can be described by the diffusion of one type of charged particles only, viz. $V_{0}{ }^{m}(m=1,2)$. The effective (i.e. measured) chemical diffusion coefficient $\tilde{D}_{v}$ for the vacancies as obtained from the conduction measurements is related to the microscopic diffusion coefficient $D_{v}$. which describes the diffusion of $V_{0}{ }^{m} \cdot$ when no gradient in chemical potential exists. The relation between $\tilde{D}_{v}$ an $D_{v}$ 


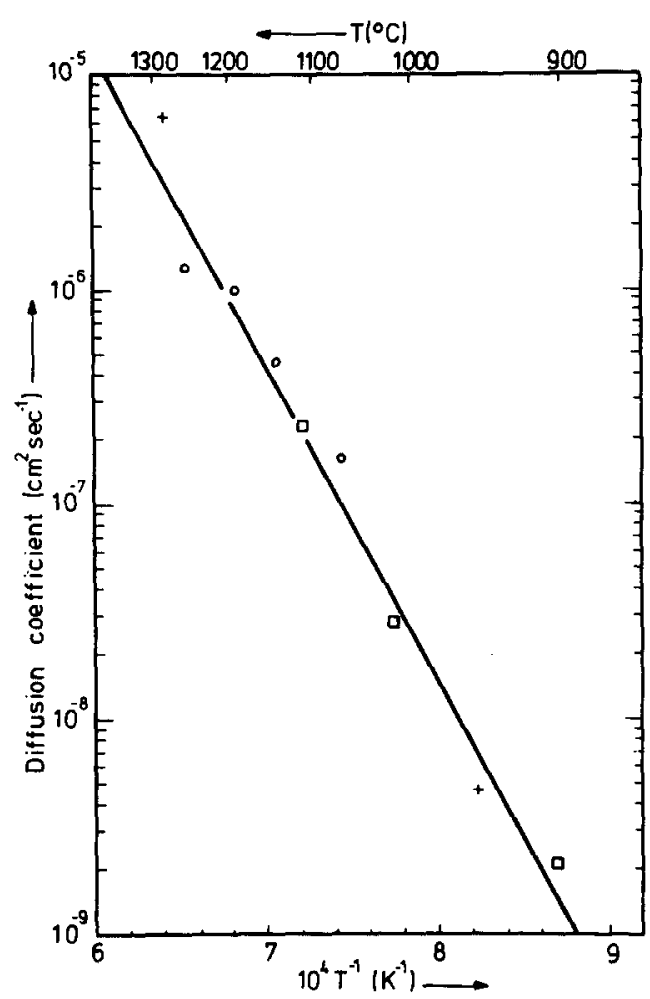

Fig. 6. Diffusion coefficient of oxygen vacancies for different types of YIG samples. $\square$, Polycrystalline Ca-doped sample; $O$, Single-crystalline Si-doped sample A (see Table 1); + , Singlecrystalline Si-doped sample B (see Table 1). Solid line: Fit of the experimental data for a single-crystalline $\mathrm{Zn}$-doped sample (see Fig. 5).

is given by [12]

$$
\begin{array}{ll}
\tilde{D}_{v}=D_{v}\left(1-m \frac{\partial \ln p}{\partial \ln \left[V_{0}^{m \cdot}\right]}\right), & p \gg n \\
\tilde{D}_{v}=D_{v}\left(1+m \frac{\partial \ln n}{\partial \ln \left[V_{0}^{m \cdot}\right]}\right), \quad n \gg p .
\end{array}
$$

The second term in the brackets arises from the counter diffusion of holes (eqn 12a) or the parallel diffusion of electrons (eqn 12b) with the diffusion of the positively charged vacancies.

Since

$$
\frac{\partial \ln p}{\partial \ln \left[V_{0}{ }^{m \cdot}\right]} \ll 0
$$

and

$$
\frac{\partial \ln n}{\partial \ln \left[V_{0}^{m \cdot}\right]} \geqslant 0
$$

$\tilde{D}_{v}$ is generally enhanced with respect to $D_{v}$. In the derivation of eqns (12) it is assumed that the vacancy diffusion is rate determining and not the hole or electron diffusion.

If the vacancy concentration is small with respect to the oxygen concentration in the lattice and if the diffusion proceeds only by interchange of $V_{0}{ }^{m}$ and oxygen ions, we have

$$
D_{v}=\alpha d_{v}^{2} \Gamma_{v} x_{0} \approx \alpha d_{v}^{2} \Gamma_{v}
$$

where $x_{0}(\approx 1)$ is the site fraction of oxygen ions, $\alpha$ is a geometrical constant, $d_{v}$ is the jump distance of the vacancy and $\Gamma_{v}$ is the jump frequency. The temperature dependence of $D_{v}$ is contained in the temperature dependence of $\Gamma_{v}$. Now $\Gamma_{v}$ can be written as $\Gamma_{v}=$ $\nu \exp \left[\left(T S_{m}-H_{m}\right) / k T\right]$, where $\nu$ is a frequency factor, $S_{m}$ is the migration entropy and $H_{m}$ the migration enthalpy of the moving species. This means that the activation enthalpy of the microscopic diffusion coefficient only contains the migration enthalpy $H_{m}$.

In order to determine $D_{v}$ from $\vec{D}_{v}$ the actual relation between $p$ (or $n$ ) and $\left[V_{0}{ }^{m}\right]$ has to be known. We have calculated $\tilde{D}_{v} / D_{v}$ for some limiting cases of the neutrality equation. For this purpose we first simplify this equation by assuming complete ionization of donors and acceptors, i.e. we write

$$
n+[A]_{\mathrm{tot}}=p+[D]_{\mathrm{tot}}+2\left[V_{0}\right] .
$$

Next we further simplify eqn (14) depending. on the relative magnitudes of the different terms. The resulting neutrality equations are given in Table 2. Assuming a formation enthalpy $H_{v}$ for the formation of $V_{0}$ according to eqn (1) we obtain a relation between $\left[V_{0}\right]$ and $n$ by rewriting eqn (3) in the form

$$
\left[V_{0}^{\prime}\right] n^{2}=K_{v} P_{\mathrm{O}_{2}}^{-1 / 2}=K_{v 0} \mathrm{e}^{-H_{\mathrm{r}} / k T} P_{\mathrm{O}_{2}}^{-1 / 2}, \quad n \geqslant p .
$$

For $p$-type materials we have, since $n p=K_{i 0}$ $\times \exp \left(-E_{i} / k T\right)$ the relation:

$$
\left[V_{0}\right] p^{-2}=K_{v 0} K_{i 0}^{-2} \mathrm{e}^{-\left(H_{v}-2 E_{i}\right) / k T} P_{O_{2}}^{-1 / 2}, \quad p \gg n .
$$

Using eqns (12)-(16) we have calculated the enhancement factor $\tilde{D}_{v} / D_{v}$ and the activation energy $\tilde{Q}$ of $\tilde{D}_{v}$. The result is presented in Table 2 .

It can be seen that only when the concentrations of donors and acceptors are of equal magnitude one finds a strong enhancement of $\tilde{D}_{v}$ and at the same time the activation energy of $\tilde{D}_{v}$ is different from $H_{m}$.

Experimentally we find that our results for the $p$-type as well as for the $n$-type samples can be described by eqn (11). With reference to Table 2 we conclude that under our experimental conditions $\tilde{D}_{v} / D_{v} \approx 1$ and that the activation enthalpy obtained from eqn (11) is the migration enthalpy, i.e. $\tilde{Q}=H_{m}=2.90 \mathrm{eV}$.

(b) The tracer and microscopic diffusion coefficients

The tracer diffusion coefficient $D^{T}$ has been measured in single crystalline and polycrystalline YIG by Paladino et al. [6], who investigated the diffusion of $\mathrm{O}^{18}$ isotopes. The results for both the single crystal and the polycrystalline samples could be described by the expression

$$
D^{T}=0.4 \exp (-2.84 \mathrm{eV} / k T) \mathrm{cm}^{2} \mathrm{~s}^{-1} .
$$

The self diffusion coefficient $D_{0}$ is given by $D_{0}=D^{T} / f$, where $f$ is a correlation factor $\sim 1$. Further $D_{0}$ is given by

$$
D_{0} \approx \alpha d_{v}^{2} \Gamma_{\nu} x_{v}=D_{x} x_{v}
$$


Table.2. Ratio of the chemical diffusion coefficient $\tilde{D}_{v}$ and the microscopic diffusion coefficient $D_{v}$ of oxygen vacancies $V_{0}$ for different neutrality equations. Also given are the activation enthalpies of $\tilde{D}_{\mathrm{v}}$ and of the self diffusion coefficient $D_{0}$ for these neutrality equations (see Section 5)

\begin{tabular}{|c|c|c|c|c|}
\hline Relative matriludes & Noutrality equation & $\widetilde{\mathrm{D}}_{\mathrm{v}} / \mathrm{D}_{\mathrm{v}}$ & $\begin{array}{c}\text { Activation enthaipy } \\
\text { of } \tilde{D}_{v}\end{array}$ & $\begin{array}{l}\text { Activation } \\
\text { entligipy } \\
\text { of } D_{0}\end{array}$ \\
\hline $\begin{array}{l}n \gg p \\
n \gg\left[v_{0} \cdot\right] \\
{[D]>[A]}\end{array}$ & $n \approx[D]-[A]$ & 1 & $\mathrm{H}_{\mathrm{m}}$ & $\mathrm{H}_{\mathrm{in}}+\mathrm{H}_{\mathrm{V}}$ \\
\hline $\begin{array}{l}n>p \\
\left.\left[v_{0}\right]>2 v\right]-[A] \\
{[D] \geqslant[A]}\end{array}$ & 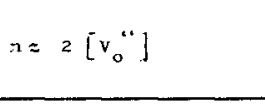 & j & $H_{m}$ & $\mathrm{H}_{\mathrm{m}}+\mathrm{H}_{\mathrm{v}} / 3$ \\
\hline 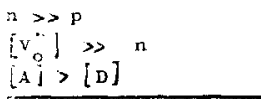 & $2\left[v_{0}^{*}\right]=[A]-[D]$ & $\left(1+2 \frac{[A]-[n]}{n}\right) \gg 1$ & $\mathrm{H}_{\mathrm{m}}+\mathrm{H}_{\sqrt{ }} / 2$ & $\mathrm{H}_{\mathrm{m}}$ \\
\hline $\begin{array}{l}p \gg n \\
{[v * 1 \gg p} \\
{[A]>[D]}\end{array}$ & $2\left[\mathrm{v}_{0}^{*}\right]=[A]-[D]$ & $\left(1+2 \frac{[A]-[D]}{p}\right)>>1$ & $\mathrm{H}_{\mathrm{m}}+\mathrm{H}_{\mathrm{v}} / 2-\mathrm{E}_{\mathrm{i}}$ & $\mathrm{H}_{\mathrm{m}}$ \\
\hline $\begin{array}{l}p \gg n \\
p \gg\left[v_{0}^{\prime}\right]\end{array}$ & $p \approx[A]-[D]$ & 1 & $\mathrm{H}_{\mathrm{m}}$ & $\mathrm{H}_{\mathrm{m}}+\mathrm{H}_{\mathrm{v}}-2 \mathrm{E}_{\mathrm{i}}$ \\
\hline
\end{tabular}

where $x_{v}$ is the site fraction of oxygen vacancies. Using this equation expressions for the activation energy of $D_{0}$ have been derived and the results are shown in the last column of Table 2. This table illustrates the following general statement concerning the self diffusion of a particle $i$. The formation enthalpy $H_{v}$ of $i$ only enters the expression for the activation energy of $D_{i}$ when the concentration $[i]$ is determined by the partial pressure of one of the constituents. In the case that $[i]$ is determined completely by the concentration of donors and/or acceptors the activation energy only contains the migration enthalpy $H_{m}$. Experimentally the tracer diffusion experiments show an activation energy of $2.84 \mathrm{eV}$ which agrees within the experimental uncertainty with the value of the migration enthalpy $H_{m}=2.9 \pm 0.1 \mathrm{eV}$ which is found from our work. We therefore may conclude that the concentration of the charged oxygen vacancies in the samples of Paladino c.s. [6] was determined by the impurity concentrations. (Case 3 or 4 of Table 2.) Using eqn (17) we can estimate the oxygen vacancy concentration in the samples used for the tracer diffusion experiments to be $x_{v}=D_{0} / D_{v} \approx 0.4 / 8400=5.0 \times 10^{-5}$, corresponding to $6.0 \times 10^{-4}$ oxygen vacancies per formula unit, or a concentration of $2 \times 10^{18} \mathrm{~cm}^{-3}$. According to Table 2 this value should be equal to the difference between the acceptor and donor concentrations. It is well known that undoped YIG can contain appreciable amounts of impurities like $\mathrm{Pb}, \mathrm{Ca}, \mathrm{Si}, \mathrm{F}$ or $\mathrm{Pt}[3]$ and the above mentioned vacancy concentration seems to be of very reasonable magnitude. It would be of great interest, however, to measure the tracer diffusion in YIG samples as a function of doping.

\section{SUMMARY}

In many compounds the concentration of native defects can be altered by changing the partial vapour pressure of a constituent at a sufficiently high temperature. Such a change in defect concentration is often accompanied by a change in electrical conductivity. We have shown that for small changes in partial pressure the changes in conduc- tivity and defect concentration are linearly related and that under these conditions the time dependence of $\sigma$ after a stepwise change in the partial pressure can be used to calculate the chemical diffusion coefficient of the defect.

More specifically we have shown that this procedure can be used to calculate the diffusion coefficient of oxygen vacancies in yttrium iron garnet in the temperature region $900-1400^{\circ} \mathrm{C}$, when the partial pressure is varied stepwise between 1 and $0.1 \mathrm{~atm}$. Measurements on both $p$-and $n$-type single crystals and on $p$-type polycrystalline YIG samples all yielded the same values of the constants in the expression for the diffusion coefficient as a function of temperature. From an analysis of the experimental data in terms of possible models relating the defect concentrations in these materials we conclude that the activation energy of the diffusion has to be attributed solely to the migration enthalpy of the oxygen vacancies. We also find a close agrecment between the temperature dependence of the diffusion coefficient of oxygen vacancies and of the tracer diffusion coefficient of $\mathrm{O}^{18}$ as determined by Paladino et al. [6].

Acknowledgements-We would like to thank W. Tolksdorf, Philips Forschungslaboratorium, Hamburg, for the single crystals and the chemical analysis. Thanks are also due to R. Wernicke and K. H. Härdtl, Philips Forschungslaboratorium, Aachen, for useful discussions.

\section{REFERENCES}

1. Larsen P. K, and Metselaar K., J. Solid State Chem. 12, 253 (1975).

2. Larsen P. K. and Robertson J. M., J. Appl. Phys. 45, 2867 (1974).

3. Metselaar R. and Huyberts M. A. H., J. Phys. Chem. Solids 34, 2257 (1973).

4. Hagedorn F. B., J. Appl. Phys. 45, 3123 (1974).

5. Metselaar R. and Larsen P. K., Solid State Commun. 15, 291 (1974).

6. Paladino A. E., Maguire E. A. Jr. and Rubin L. G., J. Am. Ceram. Soc. 47, 280 (1964).

7. Metselaar R, and Huyberts M. A. H., to be published.

8. Larsen P. K. and Metselaar R., to be published. 
9. Crank J., The mathematics of diffusion. Oxford University Press, Oxford (1956).

10. Wernicke R., private communication.

11. These single crystals and the analysis results were placed at our disposal by Tolksdorf $W$.

12. Kröger F. A., The chemistry of imperfect crystals, Vol. 3. North-Holland, Amsterdam (1974).

13. Blakemore J. S., Semiconductor statistics. Pergamon Press, Oxford (1962).

\section{APPENDIX}

We consider a partially compensated non-degenerate semiconductor containing foreign donors and acceptors of concentrations $[D]_{\mathrm{tot}}$ and $[A]_{\mathrm{tot}}$ respectively and native donors of concentration $[C]_{\text {tor }}$. For reasons of simplicity we assume that the defects can only be singly charged or neutral. While $[D]_{\text {rot }}$ and $[A]_{\text {tot }}$ are fixed $[C]_{\text {tot }}$ can be varied by some external parameters (e.g. the oxygen partial pressure). The complete neutrality equation is

$$
n+\left[A^{\prime}\right]=p+\left[D^{\prime}\right]+\left[C^{\prime}\right]
$$

where $\left[A^{\prime}\right] \leqslant[A]_{\text {lot }},\left[D^{\circ}\right] \leqslant[D]_{\text {tot }}$ and $\left[C^{\prime}\right] \leqslant[C]_{\text {tot }}$ and $n$ and $p$ are the densities of free charge carriers in the conduction and valence band respectively. The conductivity is given by $\sigma=$ $n e \mu_{-}+p e \mu_{+}$, where $\mu_{-}$and $\mu_{+}$are the mobilities of electrons and holes respectively. Near the minimum value of the conductivity, $\sigma_{\min }=2\left(N_{+} N_{-}\right)^{1 / 2}\left(\mu_{+} \mu_{-}\right)^{1 / 2} \exp \left(-E_{i} / 2 k T\right)$, where $N_{+}$and $N_{-}$are the effective densities of states of valence and conduction band and $E_{i}$ the band gap energy, $\sigma$ is not a monotonic function of $[C]_{\text {cot. }}$. This region is therefore not suitable for the diffusion measurements, and we restrict our discussion to the cases $n \gg p$ or $p \gg n$. We can further distinguish between complete and incomplete ionization of the defects.

\section{(a) Complete ionization}

In this case the concentration of ionized defects is equal to the total defect concentration and using eqn (A1) we find

$$
\sigma^{(1)}=\frac{\partial \sigma}{\partial[C]}=\mp 2 \mu_{ \pm} e
$$

where the upper sign is valid for $p \gg n$ and the lower for $n \gg p$.

For complete ionization the relationship between $\sigma$ and $C$ is thus linear.

For undoped, pure materials it is possible that $[A]_{\text {tot }}$ and $[D]_{\text {tot }}$ are negligible with respect to $[C]_{\text {tort }}$. The situation $p \gg n$ is then not possible for defects $C$ of donor type.

For $p \leqslant n$ eqn (A2) is then applicable.

\section{(b) Incomplete ionization}

We have made a calculation of the dependence $p=p\left([C]_{\text {tot }}\right)$ in the $p$-type range, $p \gg n$, under conditions comparable to those met for YIG. We assume a band gap energy of about $2 \mathrm{eV}$, a temperature of $1129^{\circ} \mathrm{C}(k T=0.12 \mathrm{eV})$ and due to the high band gap energy, a complete ionization of the donors $\left[D^{\circ}\right]=[D]_{\text {tot }}$ and $\left[C^{*}\right]=[C]_{\text {tot }} ;\left[A^{\prime}\right]$ and $p$ are determined by the Fermi energy $E_{F}, \quad\left[A^{\prime}\right]=[A]_{\text {tot }}\left[1+\beta \exp \left[\left(E_{A}-E_{F}\right) / k T\right]\right\}^{-1} \quad$ and $\quad p=$ $N_{+} \exp \left(-E_{F} / k T\right)[13]$. Here the top of the valence band is chosen as the zero of the energy scale, $E_{A}$ is the acceptor ionization energy and the degeneracy factor $\beta=2$.

Inserting $\left[A^{\prime}\right]$ and $p$ in the neutrality equation, $E_{F}$ can be calculated and subsequently $\left[A^{\prime}\right]$ and $p$ determined. The results of a calculation using $[A]_{\text {tot }}=8 \times 10^{19} \mathrm{~cm}^{-3}, N_{+}=2 \times 10^{21} \mathrm{~cm}^{-3}$ and $[D]_{\text {tot }}=2 \times 10^{18} \mathrm{~cm}^{-3}$ is shown in Fig. 7. Here $p$ is given as a function of $[C]_{\text {rot }}$ with $E_{\mathrm{A}}$ as a parameter. The deviation from the linear relationship between $p$ and $[C]_{\text {tot }}$ increases with $E_{A}$.

In Section 2(b) it was mentioned that for sufficiently small changes in $C$ an approximately linear relationship between $\sigma$ (or here $p$ ) and $C$ exists. Assuming a $10 \%$ change in the conductivity $\left[\sigma(C)-\sigma\left(C_{0}\right)\right] / \sigma\left(C_{0}\right)=0.1$ we have derived the relative change $\alpha$ in $\sigma^{(1)}(C), \alpha=\left[\sigma^{(1)}(C)-\sigma^{(1)}\left(C_{0}\right)\right] / \sigma^{(1)}\left(C_{0}\right)$.

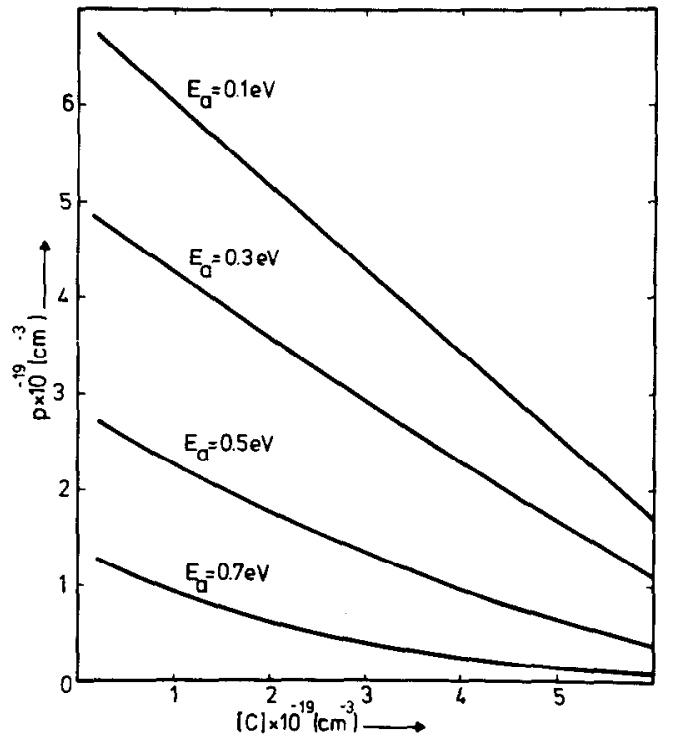

Fig. 7. Concentration of holes $p$ as a function of the defect concentration $[C]$ for a system satisfying the neutrality equation $[D]+[C]+p=\left[A^{\prime}\right]$. Concentration of extrinsic donors $[D]=$ $2 \times 10^{18} \mathrm{~cm}^{-3},\left[A^{\prime}\right]$ is the number of ionized acceptors, for a total acceptor concentration $[A]=8 \times 10^{19} \mathrm{~cm}^{-3}$, with the acceptor ionization energy $E_{A}$ as a parameter. $k T=0.12 \mathrm{eV}$, density of states in the valence band $N_{+}=2 \times 10^{21} \mathrm{~cm}^{-3}$.

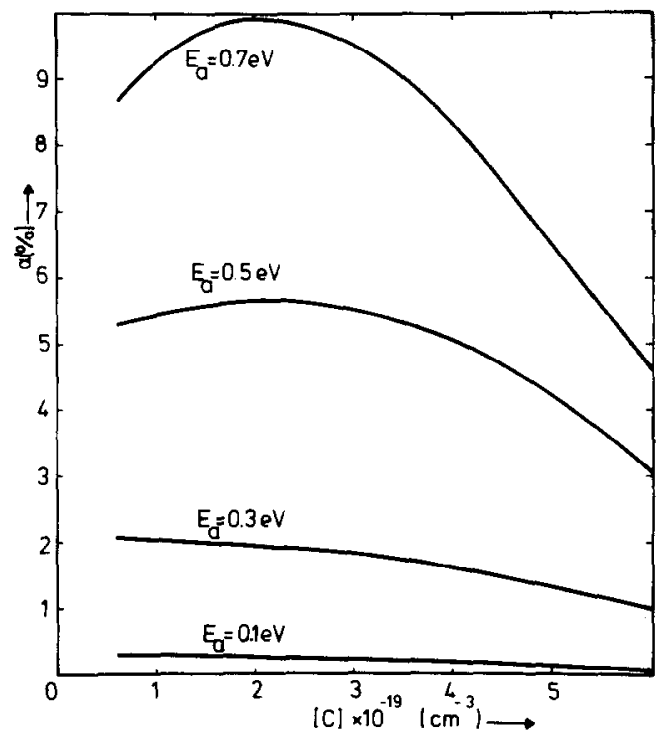

Fig. 8. The relative change $\alpha$ of $\mathrm{d}[C] / \mathrm{d} p$ as a response to a relative change in the hole concentration $\Delta p / p=0.1$, plotted as a function of the defect concentration $[C]$, with the acceptor ionization energy $E_{a}$ as a parameter. All other parameters are as specified in Fig. 7.

In Fig. $8 \alpha$ is shown as a function of $C$, again with $E_{a}$ as parameter. We see that for $E_{A} \leqslant 0.5 \mathrm{eV}, \alpha$ is smaller than 0.06 . Therefore we conclude that we can indeed use the linear approximation (eqn 7) as long as we keep the relative change in the conductivity to $10 \%$ or less.

For the $n$-type range, $n \gg p$, the calculation is quite similar and analogous conclusions can be drawn. 